\title{
Numerical Simulation of Gas Flow Field with Public Exhaust System of Shipborne Missile
}

\author{
Liang $\mathrm{Mo}^{1, \mathrm{a}}$, Jiang $\mathrm{Yi}^{2, \mathrm{~b}}$ \\ ${ }^{1} 5$ South Zhongguancun Street, Haidian District, Beijing Postcode, 100081, China \\ ${ }^{2} 5$ South Zhongguancun Street, Haidian District, Beijing Postcode, 100081, China \\ aemail:liangmo51020@163.com, bemail:kli20112012@163.com
}

Keywords: Vertically Launching; Public Exhaust System; Jet; Numerical Simulation

\begin{abstract}
The mathematical model of a vertical launching device was established by simplifying its physical model. Through the method of solving Navier-Stokes equation, the complex internal flow of the exhaust was simulated when the missile keep still in the Vertical Launching System, and the changes of the various parameters of the flow field were analyzed. A deepen understanding of the unsteady flow can be made through the analysis. The work of this paper may offer some reference and gist for research on the public exhaust system of jet flow in a vertical launching system.
\end{abstract}

\section{Introduction}

Vertical launch is a major mode of shipborne missile, and a structure with the public exhaust system in the vertical launching system on shipboard is a common structure model of vertical launch. Since the structure of the public exhaust system is relatively complex, and the vertical launch space is very compact, but a large number of high-temperature, high-speed gas jet was ejected when the missile launched, which makes the motion of the gas in the public exhaust system is caused concern. It is one of the key problems to be solved in vertical launching mode that whether the gas can be exhausted smoothly and reliable, which directly related to the success or failure of a missile launch. The three-dimensional unsteady flow flied can be clearer understanding through the numerical simulation, and it can be a guide for solving practical problems.

This paper taking a certain type of missile which keeps still in the vertical launching system as example, the Reynolds averaged N-S equations and $R N G k-\varepsilon$ two-equation turbulence model are used to simulate the three-dimensional complex flow flied of the gas exhaust system, and the distribution of temperature and pressure are presented.

\section{Fluid dynamics control equation}

When the missile was fired, high-temperature, high-speed gas-solid gas stream ejected from the engine. To simplify the calculations, the impact of solid particles in the jet was ignored. The three-dimensional Reynolds averaged Navier-Stokes equations are as follows:

Continuity equation

$\frac{\partial \rho}{\partial t}+\nabla \cdot(\rho \vec{u})=0$

Viscous momentum equation

$\frac{\partial}{\partial t}\left(\rho u_{i}\right)+\frac{\partial}{\partial x_{j}}\left(\rho u_{i} u_{j}\right)=-\frac{\partial p}{\partial x_{i}}+\frac{\partial \tau_{i j}}{\partial x_{j}}$

Energy equation

$\frac{\partial}{\partial t}(\rho H)+\frac{\partial}{\partial x_{j}}\left(\rho u_{j} H\right)=\frac{\partial p}{\partial t}+\frac{\partial}{\partial x_{j}}\left(u_{i} \tau_{i j}-q_{j}\right)$

Based on the finite volume method, RNG $k-\varepsilon$ model was chose as turbulence model. 


$$
\begin{aligned}
& \frac{\partial}{\partial t}(\rho k)+\frac{\partial}{\partial x_{i}}\left(\rho k u_{i}\right)=\frac{\partial}{\partial x_{i}}\left(\alpha_{k} \mu_{e f f} \frac{\partial k}{\partial x_{j}}\right)+G_{k}+G_{b}-\rho \varepsilon-Y_{M}+S_{k} \\
& \frac{\partial}{\partial t}(\rho \varepsilon)+\frac{\partial}{\partial x_{i}}\left(\rho \varepsilon u_{i}\right)=\frac{\partial}{\partial x_{j}}\left(\alpha_{k} \mu_{e f f} \frac{\partial \varepsilon}{\partial x_{j}}\right)+C_{1 \varepsilon} \frac{\varepsilon}{k}\left(G_{k}+C_{3 \varepsilon} G_{b}\right)-C_{2 \varepsilon} \rho \frac{\varepsilon^{2}}{k}-R_{\varepsilon}+S_{\varepsilon} \\
& R_{\varepsilon}=\frac{C_{\mu} \rho \eta^{3}\left(1-\eta / \eta_{0}\right)}{1+\beta \eta^{3}} \frac{\varepsilon^{2}}{k}
\end{aligned}
$$

\section{Physical model}

The shipborne vertical launcher uses the public exhaust system, which consist of pressure chamber, exhaust port and the missile launch canister installed in the launch position. The pressure chamber was divided into 2 rows of 8 launch positions. The launch positions were sealed by sealing cover which can be broke by jet from the side of missile launch canister. The exhaust port, which is not divided as the pressure chamber, connects with 2 rows of launch positions. In the connect place, there is a guiding plate, which can make the gas flow into the exhaust port easily and separate another row of pressure chamber when the missile launching. In the vertical launching process, the 4 pressure chamber, exhaust port and the missile launch canister installed in the launch position composed of gas exhaust system, which can exhaust the gas flow out of the launching device.

The jet has a greater impact on the bottom of the gas exhaust system when the missile keep still in the process of launch; it is harder for the 4 outside launch positions to exhaust the gas. In this paper, after establishing the simplified fluid model, the flow field of the outside launch position was calculated when the missile keep still in the Vertical Launching System. The gas was made the following assumptions:

1) The gas flow is inviscid, incompressible ideal gas;

2) No chemical reaction and heat losses was considered;

3) The gas flow was considered as unsteady flow of two-phase equilibrium flow.

To ensure the accuracy of calculation, the flow field was mostly divided by structured grid because of the complexity of the model. The calculation model and the local grid are shown in figure 1.
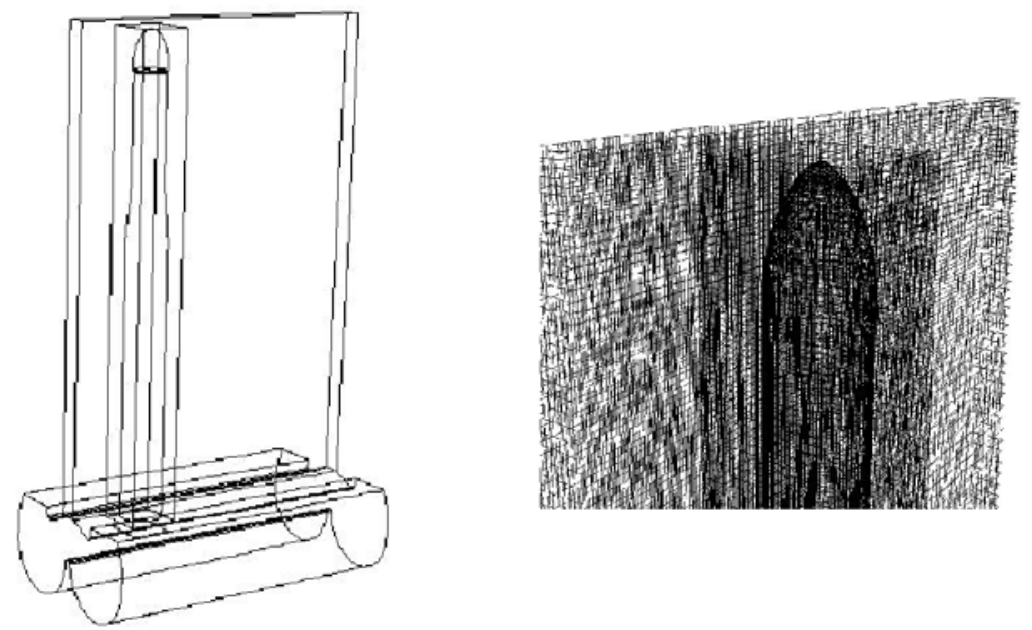

Fig.1. Calculation model and the local grid

\section{Result analysis}

The flow field of the outside launch position was calculated when the missile keep still in the Vertical Launching System after importing the model into Fluent and Setting the boundary conditions. The results are as follows when the calculation of the flow field is stable.

The pressure contours in pressure chamber is shown in Fig.2. High pressure areas centralize in the wall of pressure chamber just blow missile nozzle. After the high-pressure jet impinging the 
bottom surface of pressure chamber, the pressure decreased rapidly. The high-pressure jet has little impact on lunch position nearby because of the structure of launching device.

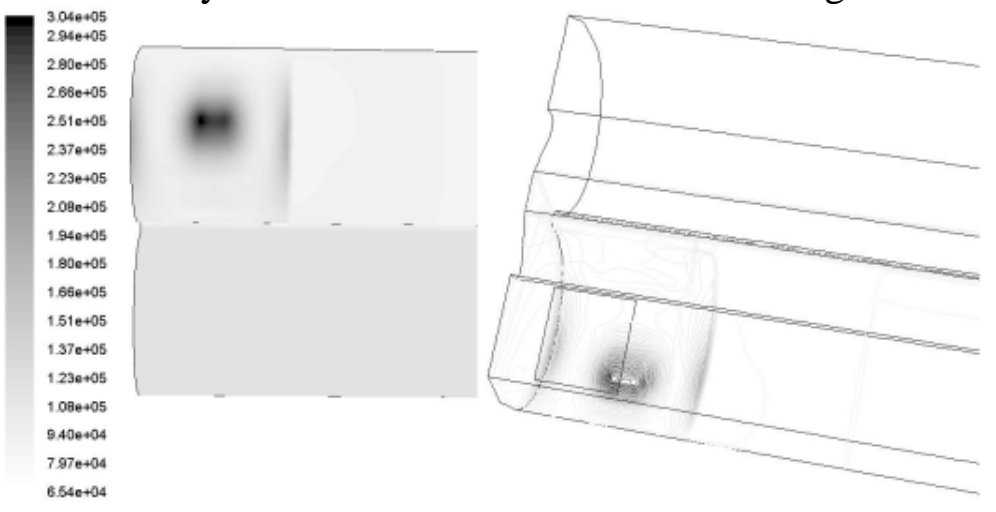

Fig.2. Calculation model and the local grid

The temperature contours in the whole flow field is shown in Fig.3. Overall, most of the high-temperature gas flows from the pressure chamber and exhaust port, and a small part from the missile launch canister. The high temperature gas has smaller effect of ablation on the missile, and the highest temperature (2650K) appears in the lower part of the missile. The highest temperature of launch position besides the launching one which has much more high-temperature influence, appears nearby the sealing cover, and it is about $1020 \mathrm{~K}$. The high temperature area of the exhaust port is where the high-temperature pressure chamber faces to, and with the increasing of gas diffusion, the impact area of the upper part becomes bigger.
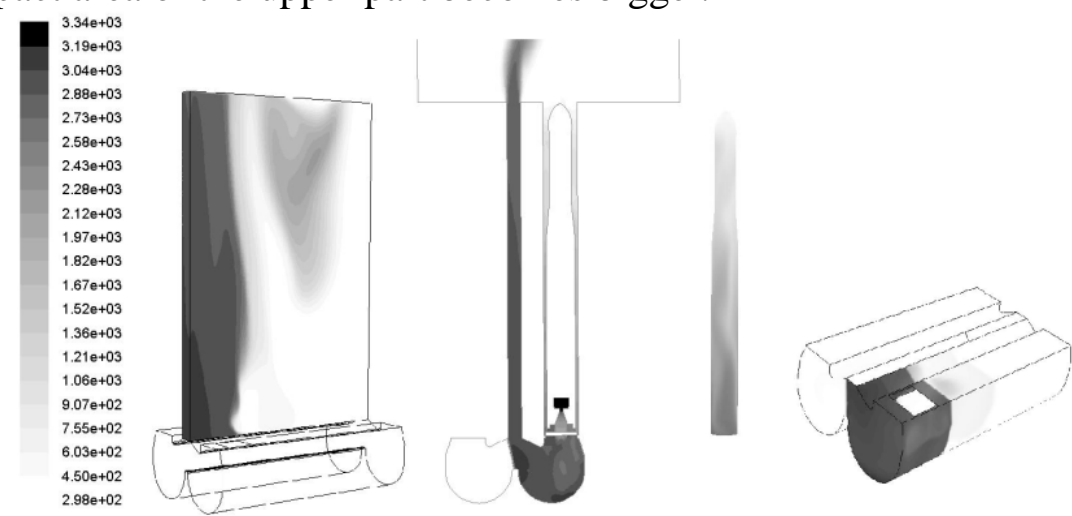

Fig.3. Temperature contours in the whole flow field

The velocity vector of different time in the pressure chamber is shown in Fig.4. It can be see that with the movement of jet, the vortex will be formed on both sides of jet, and the vortex will be gradual downward with the movement of jet. When the jet reached the bottom of pressure chamber, the vortex has also been gradually pushed close to the bottom. With the flow of gas, the vortex in the side of exhaust port was not disappeared, but kept still at the bottom of pressure chamber.

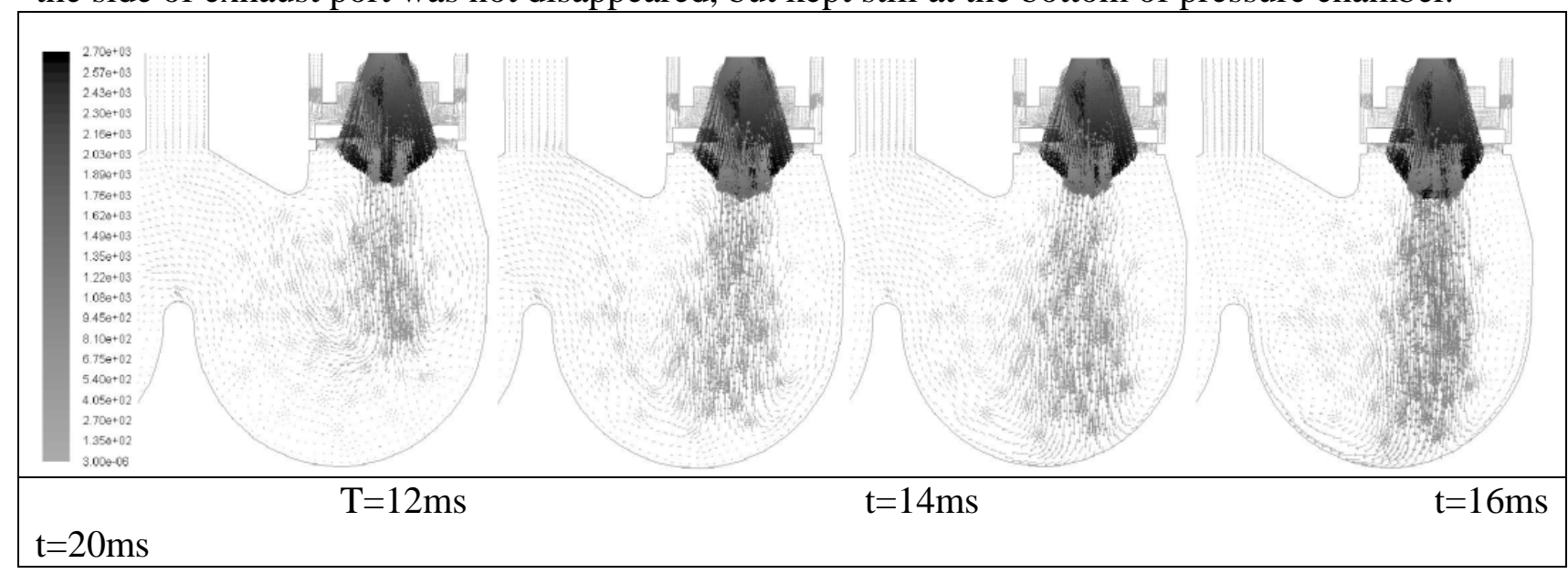

Fig.4. Velocity vector of different time in the pressure chamber 
The velocity vector of different time in the exhaust port is shown in Fig.5. The velocity of gas in the exhaust port is increasing over time, but there is no vortex separation with the change of pressure. Gas can go through the exhaust port smoothly. It also shows the gas pressure has been greatly reduced when flowing into the exhaust port, and was close to atmospheric pressure.

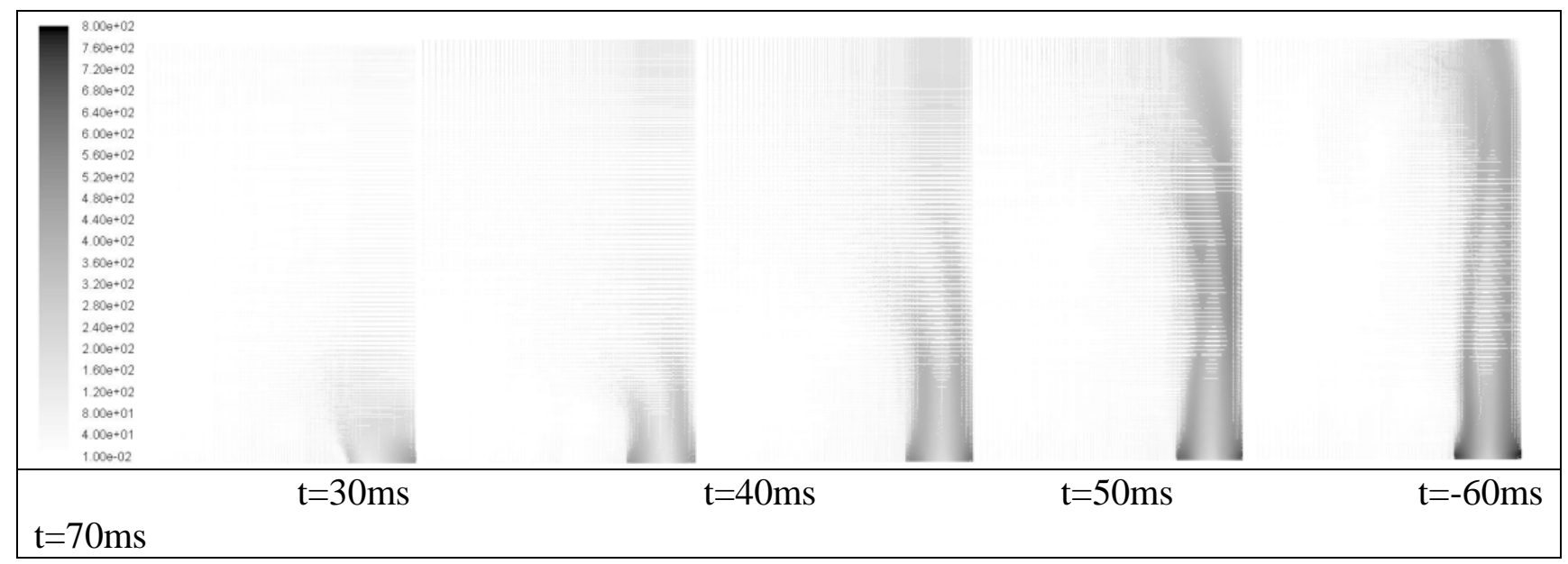

Fig.5. Velocity vector of different time in the exhaust port

\section{Conclusion}

The calculation results show that: the numerical simulation can simulate the real flow process of gas in the vertical launching device with the public exhaust system, show the characteristics of gas flow field distribution, provide the basis for design of vertical launching device. The vortex formed in the pressure chamber and its steady state will hinder the exhaust of gas, increase the pressure of pressure chamber. The gas can flow smoothly in the exhaust port, but the utilization ratio of the exhaust port is low. The improvements should be took in the design of the public exhaust system to increase the utilization ratio of the exhaust port and reduce the effect of temperature to the wall of exhaust port.

\section{References}

[1] Armsfield Sand Stre R. Thefractional-step method for the Navier-Stokes equations on staggered grids: accuracy of three variations[J]. Journal of Computational Physics, 153:660-665, 1999.

[2] ZHAO Cheng-qing and JIANG Yi: Gas Jet Dynamics (Beijing Institute of Technology Press, Beijing, China 1998).

[3] J.D. Anderson: The Basis with Applications of Computational Fluid Dynamics (McGraw-Hill Companies, Inc. 1995).

[4] WU Li-min: Numerical Simulation of the Internal Flow in the Vertical Launching System, Ship Science and Technology.2003, 25(2).

[5] WU Li-min, CHEN Jin-cao: Research on the Exhaust Jet in the Exhaust Channel of the Vertical Launching System, Ship Science and Technology.2007, 29(Supplement 1). 